\title{
A novel adaptive active noise control algorithm based on Tikhonov regularisation
}

\author{
Iman Ardekani ${ }^{1}$, Neda Sakhaee ${ }^{2}$, and Hamid Sharifzadeh, Bashar Barmada, Gerard Lovell ${ }^{3}$ \\ ${ }^{1,3}$ Unitec Institute of Technology, Auckland, New Zealand \\ ${ }^{2}$ The University of Auckland, Auckland, New Zealand
}

\begin{abstract}
This paper proposes a novel adaptive active noise control algorithm based on Tikhonov regularization theory. A regularized cost function consisting of the weighted sum of the most recent samples of the residual noise and its derivative is defined. By setting the gradient vector of the cost function to zero, an optimal solution for the control parameters is obtained. Based on the proposed optimal solution, a computationally efficient algorithm for adaptive adjustment of the control parameters is developed. It is shown that the regularized affine projection algorithm can be considered as a very special case of the proposed algorithm. Different computer simulation experiments show the validity and efficiency of the proposed algorithm.
\end{abstract}

Keywords: active noise control, adaptive algorithms, Tikhonov regularization, affine projection algorithm

\section{INTRODUCTION}

Active Noise Control (ANC) systems use the principle of destructive superposition of sound fields to create local zones of silence. ${ }^{1}$ Figure 1 shows a generic block diagram for single channel ANC systems. In this figure, $\mathbf{p}$ and $\mathbf{s}$ represent primary and secondary paths, and $x(n)$ represents the reference signal, measured by a microphone located close to the noise source. It is assumed that the noise signal received at the desired zone of silence, $d(n)$, is the response of the primary path to the reference signal; hence, $\mathbf{p}$ can be considered as a hypothetical unknown system. The control system is responsible for generating the control signal $y(n)$ which is fed to a loudspeaker. The loudspeaker produces an anti-noise field in the environment. The control system parameters should be adjusted in such a way that the residual noise $e(n)$ which is the combination of the anti-noise and noise signals received at the desired zone of silence is minimal (in terms of signal power). Usually, a microphone called the error microphone, is located at the zone of silence to measure the residual noise.

As seen in Figure 1, an ANC control system consists of an adjustable digital filter $\mathbf{w}$ coupled with an adaptive algorithm. This digital filter, which is called the ANC controller, is usually a standard Finite-duration Impulse Response (FIR) filter. In ANC systems, the digital filter cannot be adjusted by standard adaptive algorithms e.g. Least Mean Square (LMS) or Recursive Least Square (RLS) due to the existence of the secondary path. However, the influence of the secondary path on the performance of any standard adaptive algorithm can be compensated for if the reference signal is filtered using an estimate of the secondary path. This compensation mechanism results in a new range of adaptive algorithms referred to as filtered-x adaptive algorithms, the basis of which is the Filtered-x LMS (FxLMS). ${ }^{2}$ Other versions of the FxLMS such as Filtered-x Normalised LMS $($ FxNLMS $),{ }^{3}$ Leaky FxLMS, ${ }^{4}$ Modified FxLMS (MFxLMS) ${ }^{5}$ and Filtered-weight FxLMS (FwFxLMS $)^{6}$ have been proposed to improve the performance of the original algorithm. However, a common drawback of FxLMS based adaptive algorithms is their slow convergence rate. ${ }^{7}$ To overcome this problem, more complicated algorithms such as Filtered-x Recursive Least Square $(\mathrm{FxRLS})^{8}$ or Filtered-x Affine Projection $(\mathrm{FxAP})^{9}$ can be used. These algorithms have relatively fast convergence rate; however their real-time realizations may not be cost effective. Another common drawback of these algorithms is their inefficiency when non-stationary components such as impulses and spikes appear in the noise to be canceled.

In this paper, a novel adaptive ANC algorithm which maintains its stability in the presence of non-stationary noise components is developed. Section 2 develops a regularized model for residual noise based on Tikhonov regularization theory and derived an optimal solution for the control system parameters. Section 3 develops an adaptive algorithm for the realization of the derived optimal solution. Section 4 discusses computer simulations. Finally, Section 5 gives concluding remarks. 


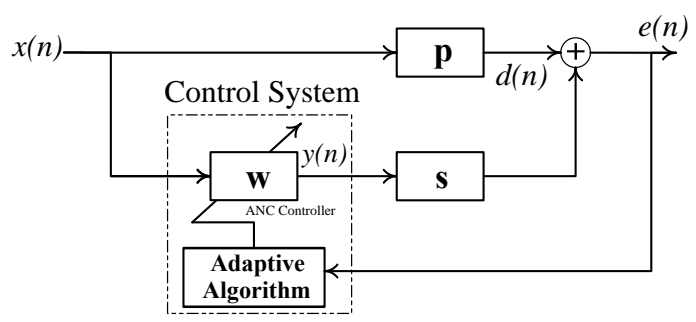

Figure 1: block diagram of single channel ANC systems

\section{A REGULARIZED SOLUTION FOR ANC CONTROLLER}

Let us define an error vector by using $M$ recent samples of the residual noise:

$$
\mathbf{e}=\left[\begin{array}{llll}
e(n) & e(n-1) & \ldots & e(n-M+1)
\end{array}\right]^{T}
$$

From the diagram, $e(n)$ can be modeled by

$$
e(n)=d(n)+\sum_{q=0}^{Q-1} \sum_{l=0}^{L-1} s_{q} w_{l} x(n-q-l)
$$

where $w_{0}, \ldots, w_{L-1}$ are the impulse response coefficients (control parameters) of the adaptive filter $\mathbf{w}$ and $s_{0}, \ldots, s_{Q-1}$ are the impulse response coefficients of the secondary path. The error vector can be modeled by combining Eqs. (1) and (2) as

$$
\mathbf{e}=\mathbf{X}_{\mathbf{s}}^{T} \mathbf{w}+\mathbf{d}
$$

where $\mathbf{w}=\left[w_{0}, \ldots, w_{L-1}\right]^{T}$ is the weigh vector, $\mathbf{d}$ contains $M$ recent samples of $d(n)$ and $\mathbf{X}_{s}$ is given by

$$
\begin{gathered}
\mathbf{X}_{s}=\left[x_{s}(n-i-j+2)\right]_{L \times M}, i=1, \ldots, L \quad j=1, \ldots, M \\
x_{s}(n)=\sum_{q=0}^{Q-1} s_{q} x(n-q)
\end{gathered}
$$

Initially, let us consider a simple cost function in the form of

$$
J(n)=\|\mathbf{\Gamma} \mathbf{e}\|^{2}=\left\|\mathbf{\Gamma}\left(\mathbf{X}_{s}^{T} \mathbf{w}+\mathbf{d}\right)\right\|^{2}
$$

where $\boldsymbol{\Gamma}$ is a $M \times M$ matrix. An optimal solution for the $\mathbf{w}$ can be obtained by solving $\nabla J(n)=0$ :

$$
\nabla J(n)=0 \Rightarrow 2 \mathbf{X}_{s} \boldsymbol{\Gamma}^{T} \boldsymbol{\Gamma}\left(\mathbf{X}_{s}^{T} \mathbf{w}+\mathbf{d}\right)=0 \Rightarrow \mathbf{w}_{\text {opt }}=-\left(\mathbf{X}_{s} \boldsymbol{\Gamma}^{T} \boldsymbol{\Gamma} \mathbf{X}_{s}^{T}\right)^{-1} \mathbf{X}_{s} \boldsymbol{\Gamma}^{T} \boldsymbol{\Gamma} \mathbf{d}
$$

Realization of $\mathbf{w}_{\text {opt }}$ given in Eq. (7) is not feasible as $d(n)$ is not available through measurement. Moreover, $\mathbf{X}_{s} \boldsymbol{\Gamma}^{T} \boldsymbol{\Gamma} \mathbf{X}_{s}^{T}$ is not invertible when $L>M$. Alternatively, a gradient based recursive algorithm in the form of $\mathbf{w}=\mathbf{w}_{p r}-\frac{\mu}{2} \nabla J(n)$ can be used, where $\mu$ is the step-size and $\mathbf{w}_{p r}$ is the previous value of $\mathbf{w}$. This algorithm can be implemented by using $\nabla J(n)=2 \mathbf{X}_{s} \boldsymbol{\Gamma}^{T} \boldsymbol{\Gamma}\left(\mathbf{X}_{s}^{T} \mathbf{w}+\mathbf{d}\right)=2 \mathbf{X}_{s} \boldsymbol{\Gamma}^{T} \boldsymbol{\Gamma e}$. In the simplest case where $\boldsymbol{\Gamma}$ is equal to the identity matrix and the error vector contains only the most recent sample of the residual noise, the gradient based recursive algorithm becomes $\mathbf{w}=\mathbf{w}_{p r}-\mu \mathbf{X}_{s} e(n)$ which is identical to the FxLMS algorithm. FxLMS is a computationally-efficient algorithm; however, it suffers from slow convergence rate and sensitivity to any non-stationary noise components. ${ }^{10,11}$ To overcome this weakness, we propose to include the derivative of the residual noise in $J(n)$ :

$$
J(n)=\|\mathbf{\Gamma e}\|^{2}+\lambda\|\mathbf{\Gamma} \triangle \mathbf{e}\|^{2}
$$

where $\lambda$ is a scalar parameter. Assuming that $x(n)$ and $d(n)$ change slowly (compared to sampling frequency), $\triangle \mathbf{e}$ is approximately proportional to $\mathbf{X}_{s}^{T} \mathbf{w}-\mathbf{X}_{s}^{T} \mathbf{w}_{p r}$. In this case, Eq. (8) can be expanded to

$$
J(n)=\left\|\mathbf{\Gamma}\left(\mathbf{X}_{s}^{T} \mathbf{w}+\mathbf{d}\right)\right\|^{2}+\lambda\left\|\mathbf{\Gamma} \mathbf{X}_{s}^{T}\left(\mathbf{w}-\mathbf{w}_{p r}\right)\right\|^{2}
$$


which leads to the following formulation for $\nabla J(n)$ :

$$
\nabla J(n)=2 \mathbf{X}_{s} \boldsymbol{\Gamma}^{T} \boldsymbol{\Gamma}\left(\mathbf{X}_{s}^{T} \mathbf{w}+\mathbf{d}\right)+2 \lambda \mathbf{X}_{s} \boldsymbol{\Gamma}^{T} \boldsymbol{\Gamma} \mathbf{X}_{s}^{T}\left(\mathbf{w}-\mathbf{w}_{p r}\right)=2 \mathbf{X}_{s} \boldsymbol{\Gamma}^{T} \boldsymbol{\Gamma e}+2 \lambda \mathbf{X}_{s} \boldsymbol{\Gamma}^{T} \boldsymbol{\Gamma} \mathbf{X}_{s}^{T}\left(\mathbf{w}-\mathbf{w}_{p r}\right)
$$

$\nabla J(n)=0$, results in an optimal solution for $\mathbf{w}$ in the form of

$$
\mathbf{w}_{o p t}=\mathbf{w}_{p r}-\left(\lambda \mathbf{X}_{s} \boldsymbol{\Gamma}^{T} \boldsymbol{\Gamma} \mathbf{X}_{s}^{T}\right)^{-1} \mathbf{X}_{s} \boldsymbol{\Gamma}^{T} \boldsymbol{\Gamma e}
$$

This solution can be realized only when $M>L$; otherwise $\mathbf{X}_{s} \boldsymbol{\Gamma}^{T} \boldsymbol{\Gamma} \mathbf{X}_{s}^{T}$ is not invertible. In practice, ANC requires a high order FIR filter (large $L$ ); therefore, usually $M<L$ which means $\mathbf{X}_{s} \boldsymbol{\Gamma}^{T} \boldsymbol{\Gamma} \mathbf{X}_{s}^{T}$ is not invertible. In order to solve this problem, we propose to add a Tikhonov regularization term to $J(n)$ :

$$
J(n)=\|\mathbf{\Gamma} \mathbf{e}\|^{2}+\lambda\|\mathbf{\Gamma} \triangle \mathbf{e}\|^{2}+\gamma\|\triangle \mathbf{w}\|^{2}
$$

where $\gamma$ is the regularization parameter. This idea was proposed by Tikhonov for regularization of ill-posed problems in mathematics. ${ }^{12}$ Eq. (12) can be expanded to

$$
J(n)=\left\|\mathbf{\Gamma}\left(\mathbf{X}_{s}^{T} \mathbf{w}+\mathbf{d}\right)\right\|^{2}+\lambda\left\|\boldsymbol{\Gamma} \mathbf{X}_{s}^{T}\left(\mathbf{w}-\mathbf{w}_{p r}\right)\right\|^{2}+\gamma\left\|\mathbf{w}-\mathbf{w}_{p r}\right\|^{2}
$$

which leads to

$$
\nabla J(n)=2 \mathbf{X}_{s} \boldsymbol{\Gamma}^{T} \boldsymbol{\Gamma e}+2 \lambda \mathbf{X}_{s} \boldsymbol{\Gamma}^{T} \boldsymbol{\Gamma} \mathbf{X}_{s}^{T}\left(\mathbf{w}-\mathbf{w}_{p r}\right)+2 \gamma\left(\mathbf{w}-\mathbf{w}_{p r}\right)
$$

An optimal solution for the weight vector $\mathbf{w}$ is then obtained by solving $\nabla J(n)=0$,

$$
\mathbf{w}_{\text {opt }}=\mathbf{w}_{p r}-\left(\gamma \mathbf{I}_{L}+\lambda \mathbf{X}_{s} \boldsymbol{\Gamma}^{T} \boldsymbol{\Gamma} \mathbf{X}_{s}^{T}\right)^{-1} \mathbf{X}_{s} \boldsymbol{\Gamma}^{T} \boldsymbol{\Gamma e}
$$

where $\mathbf{I}_{L}$ denotes the identity matrix of order $L$. The $L \times L$ matrix $\lambda \mathbf{X}_{s} \boldsymbol{\Gamma}^{T} \boldsymbol{\Gamma} \mathbf{X}_{s}^{T}+\gamma \mathbf{I}_{L}$ is invertible for some values of $\gamma$ even if $M<L$.

\section{PROPOSED ADAPTIVE ANC ALGORITHM}

The optimal solution given in Eq.(15) can be realized by using a recursive equation in the form of

$$
\mathbf{w}(n)=\mathbf{w}(n-1)-\left(\gamma \mathbf{I}_{L}+\lambda \mathbf{X}_{s} \boldsymbol{\Gamma}^{T} \boldsymbol{\Gamma} \mathbf{X}_{s}^{T}\right)^{-1} \mathbf{X}_{s} \boldsymbol{\Gamma}^{T} \mathbf{\Gamma e}
$$

Eq. (16) requires inversion of a $L \times L$ matrix. In the following an alternative formulation for Eq. (16) with lower computational complexity is developed. This process starts by re-writing Eq. (16) as

$$
\mathbf{w}(n)=\mathbf{w}(n-1)-\left(\gamma \mathbf{I}_{L}+\lambda \mathbf{Z}_{s} \mathbf{Z}_{s}^{T}\right)^{-1} \mathbf{Z}_{s} \boldsymbol{\Gamma} \mathbf{e}
$$

where $\mathbf{Z}_{s}$ is defined by

$$
\mathbf{Z}_{s}=\mathbf{X}_{s} \Gamma^{T}
$$

Infinite summation of geometric series of a matrix $\mathbf{M}$ converges to $(\mathbf{I}-\mathbf{M})^{-1}$ provided that all the eigenvalues of $\mathbf{M}$ are between -1 and 1 . Thus, by setting $\mathbf{M}=-\frac{\lambda}{\gamma} \mathbf{Z}_{s} \mathbf{Z}_{s}^{T}$, one can modify Eq. (17) to

$$
\mathbf{w}(n)=\mathbf{w}(n-1)-\frac{1}{\gamma} \sum_{k=0}^{\infty}\left(-\frac{\lambda}{\gamma} \mathbf{Z}_{s} \mathbf{Z}_{s}^{T}\right)^{k} \mathbf{Z}_{s} \boldsymbol{\Gamma} \mathbf{e}
$$

Since $\left(\mathbf{Z}_{s} \mathbf{Z}_{s}^{T}\right)^{k}=\mathbf{Z}_{s}\left(\mathbf{Z}_{s}^{T} \mathbf{Z}_{s}\right)^{k-1} \mathbf{Z}_{s}^{T}$ for $k=1,2, \ldots ;$ Eq. (19) leads to

$$
\mathbf{w}(n)=\mathbf{w}(n-1)-\frac{1}{\gamma}\left\{\mathbf{Z}_{s} \boldsymbol{\Gamma} \mathbf{e}+\mathbf{Z}_{s} \sum_{k=1}^{\infty}\left(-\frac{\lambda}{\gamma} \mathbf{Z}_{s}^{T} \mathbf{Z}_{s}\right)^{k} \boldsymbol{\Gamma e}\right\}
$$




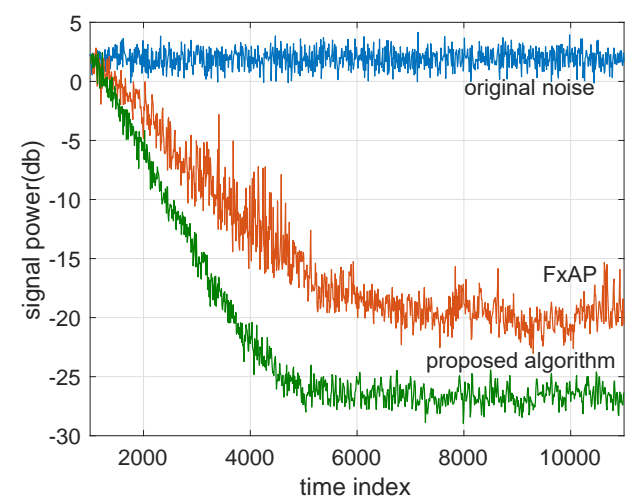

(a) with stationary noise

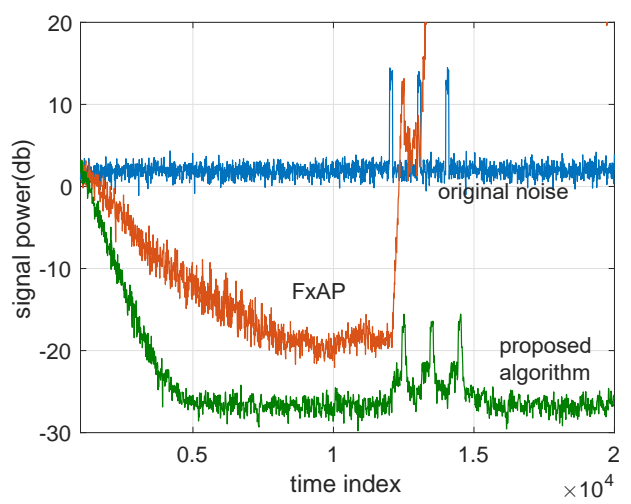

(b) with non-stationary components

Figure 2: Comparison of the proposed algorithm and FxAP: blue = original noise, red: residual noise by Fx-AP and green $=$ residual noise by the proposed algorithm

The summation of the geometric series $\sum_{k=1}^{\infty}\left(-\frac{\lambda}{\gamma} \mathbf{Z}_{s}^{T} \mathbf{Z}_{s}\right)^{k}$ can be calculated by $\left(\mathbf{I}_{M}+\frac{\lambda}{\gamma} \mathbf{Z}_{s}^{T} \mathbf{Z}_{s}\right)^{-1}-\mathbf{I}_{M}$; thus,

$$
\mathbf{w}(n)=\mathbf{w}(n-1)-\frac{1}{\gamma}\left\{\mathbf{Z}_{s} \boldsymbol{\Gamma} \mathbf{e}+\mathbf{Z}_{s}\left[\left(\mathbf{I}_{M}+\frac{\lambda}{\gamma} \mathbf{Z}_{s}^{T} \mathbf{Z}_{s}\right)^{-1}-\mathbf{I}_{M}\right] \mathbf{\Gamma e}\right\}
$$

which can be re-written by substituting $\mathbf{Z}_{s}=\mathbf{X}_{s} \boldsymbol{\Gamma}^{T}$ to

$$
\mathbf{w}(n)=\mathbf{w}(n-1)-\mathbf{X}_{s} \boldsymbol{\Gamma}^{T}\left(\gamma \mathbf{I}_{M}+\lambda \boldsymbol{\Gamma} \mathbf{X}_{s}^{T} \mathbf{X}_{s} \boldsymbol{\Gamma}^{T}\right)^{-1} \boldsymbol{\Gamma} \mathbf{e}
$$

Eq. (16) requires the inversion of a $L \times L$ matrix but Eq. (22) requires the inversion of a $M \times M$ matrix. Since $M<L$, Eq. (22) is more computationally efficient than Eq. (16). In this paper, we propose

$$
\boldsymbol{\Gamma}=\operatorname{diag}\left(r^{0}, r^{1}, \ldots, r^{M-1}\right)
$$

where $r$ is a scalar between 0 and 1. By setting $\boldsymbol{\Gamma}=\mathbf{I}_{M}$ and $\lambda=1$, Eq. (22) is simplified to the regularized FxAP algorithm:

$$
\mathbf{w}(n)=\mathbf{w}(n-1)-\mathbf{X}_{s}\left(\gamma \mathbf{I}_{M}+\mathbf{X}_{s}^{T} \mathbf{X}_{s}\right)^{-1} \boldsymbol{\Gamma} \mathbf{e}
$$

Therefore, FxAP which is a high performance ANC algorithm can be considered as an special case of the proposed ANC algorithm.

\section{COMPUTER SIMULATION}

This section discusses the effectiveness of the proposed ANC algorithm by using computer simulation. The primary and secondary paths used in the computer simulation are measured from an experimental acoustic duct reported in one of the author's previous work. ${ }^{13}$ The FIR filter used as the ANC controller is of order $L=128$. A sequence of random numbers generated by a computer is used as the primary noise. In the first experiment, the performance of the proposed ANC algorithm is compared with that of regularized Fx-AP algorithm. The regularization parameter is set to $\gamma=0.50$ for both the algorithms and $r$ is set to 0.50 for the proposed algorithm. Figure $2 \mathrm{a}$ shows the power of the residual noise signals obtained from this experiment. According to this figure, the steady state performance of the proposed algorithm is higher than that of Fx-AP. Moreover, the convergence rate of the proposed algorithm is faster than that of Fx-AP.

In the second experiment, the regularization parameter is reduced to $\gamma=0.40$ and three non stationary impulses are added to the input noise at time indices 12000, 13000 and 14000. The power of the original noise is shown by using a blue line in Figure 2a. As seen in this figure, the Fx-AP cannot maintain its stability when the impulses appear in the noise signal. However, the proposed algorithm is able to maintain its converge shortly after each impulse noise appears. 


\section{CONCLUSION}

Usually, ANC controllers are derived through minimization of the residual noise; this causes ANC systems to be highly sensitive to non-stationary characteristic of the noise to be canceled. By including the derivative of the residual noise in the ANC optimization problem, more robust ANC controllers can be obtained. However, the new optimization problem may result in unstable solutions due to its mathematical ill-posedness. The stability of the solution can be improved by adding a Tikhonov regularization term to the cost function used in the ANC optimization problem. A special case of the ANC controller obtained by the proposed method is the well-known FxAP algorithm; however, the proposed algorithm is superior to FxAP algorithm in terms of steady state performance and convergence rate.

\section{REFERENCES}

[1] S. M. Kuo and D. R. Morgan, Active noise control systems: algorithms and DSP implementations. New York, NY, USA: Wiley Interscience, 1996. 1

[2] J. C. Burgess, "Active adaptive sound control in a duct: computer simulation," Journal of the Acoustical Society of America, vol. 70, pp. 715-726, 1981. 1

[3] G. E. Warnaka, L. A. Poole, and J. Tichy, "Active acoustic attenuators," U.S. Patent 4473906, 1984. 1

[4] S. Elliott, I. M. Stothers, and P. A. Neslson, "A multiple error LMS algorithm and its applications to active control of sound and vibration," IEEE Transactions on Acoustic, Speech and Signal Processing Processing, vol. 35, pp. 1423-1434, 1987. 1

[5] M. Rupp and A. Sayed, "Two variants of the FxLMS algorithm," IEEE ASSP Workshop on Applications of Signal Processing to Audio and Acoustics, pp. 123 -126, Oct. 1995. 1

[6] I. T. Ardekani and W. H. Abdulla, "Filtered weight FxLMS adaptation algorithm: Analysis, design and implementation," International Journal of Adaptive Control and Signal Processing, vol. 25, no. 11, pp. 1023-1037, 2011. 1

[7] I. T. Ardekani and W. Abdulla, "Stochastic modelling and analysis of filtered-x least-mean-square adaptation algorithm," Signal Processing, IET, vol. 7, no. 6, pp. 486-496, August 2013. 1

[8] A. Oppenheim, E. Weinstein, K. Zangi, M. Feder, and D. Gauger, "Single-sensor active noise cancellation," IEEE Transactions on Speech and Audio Processing, vol. 2, no. 2, pp. 285 -290, Apr. 1994. 1

[9] A. Gonzalez, M. Ferrer, M. de Diego, and G. Pinero, "Fast filtered-x affine projection algorithm for active noise control," IEEE Workshop on Applications of Signal Processing to Audio and Acoustics, pp. 162-165, Oct. 2005. 1

[10] I. T. Ardekani and W. H. Abdulla, "FxLMS-based active noise control: a quick review," Proceedings of 2011 Asia Pacific Signal and Information Processing Association Annual (APSIPA) Summit and Conference, Xi'an, China, 2011. 2

[11] _ - "Stability of residual acoustic noise variance in active control of stochastic noise," in Acoustics, Speech and Signal Processing (ICASSP), 2013 IEEE International Conference on, May 2013, pp. 379-382. 2

[12] A. N. Tikhonov, "Solution of incorrectly formulated problems and the regularization method," Soviet Mathematics Doklady, vol. 4, pp. 1035-1038, 1963. 2

[13] I. T. Ardekani, "Stability analysis of adaptation process in FxLMS-based active noise control," PhD Thesis, The University of Auckland, New Zealand, 2012. 4 